\title{
Assessing the Involvement of Platelet Degranulation in the Therapeutic Properties of Exosome Derived from Amniotic Epithelial Cells through Enrichment and Interaction Network Analysis
}

\author{
M. Valizadeh ${ }^{1}$, A. Haider Bangash ${ }^{2}$, D. Hayati ${ }^{1}$, A. Jafari ${ }^{3}$, H. Rajabi-Maham ${ }^{1}$ \\ ${ }^{1}$ Faculty of Life Sciences and Biotechnology, Shahid Beheshti University, Tehran, Iran \\ ${ }^{2}$ Shifa College of Medicine, STMU, Pakistan \\ ${ }^{3}$ Student Research Committee, School of Medicine, Shahid Beheshti University of Medical Sciences, Tehran, Iran. \\ Corresponding author: Hassan Rajabi-maham, Velenjak, Tehran, Iran \\ H_Rajabi@sbu.ac.ir
}

\section{Abstract}

Platelet degranulation allows the release of large secretable pools of biologically active proteins which are critical in wound healing initiation and angiogenesis. Exosomes, which can transport a diverse suite of macromolecules, derived from amniotic epithelial cells (AEC-Exo) improve wound healing and angiogenesis. However, the underlying mechanisms are still unclear. In this investigation, we performed a user-friendly bioinformatics analysis system to identify association among the angiogenic and wound healing effects of AEC-Exo treatments. To this end, FunRich software was used, and linked to the Universal Protein Resource (UniProt) as a background database. Several enrichment analyses, including biological process, cellular component, molecular function, and protein domains were conducted on AEC-Exo proteome. Furthermore, to identify the proteins involved in platelet degranulation and evaluate protein-protein association information, comparative analyses and interaction network analyses were illustrated using the NCBI BioSystems, ExoCarta, and STRING databases. Our results indicated the statistically significant association between the proteome in AECExo, platelet degranulation, and their corresponding processes. Therefore, the involvement of platelet degranulation in AEC-Exo proteins may elucidate the angiogenic and wound-healing effects of AECExo treatments.

Keywords: Angiogenesis, Platelet degranulation, Extracellular vesicles, Enrichment analysis, Proteomics 


\section{Introduction}

The wound-healing process is a complex mechanism that requires angiogenesis as a process in which new blood vessels are formed from pre-existing ones (1). At the site of injury, platelets are essential in regulating angiogenesis but their role in repairing damaged vessels during the wound healing process is still not well known (2). Platelet degranulation allows the release of large secretable substances that promote tissue repair, angiogenesis, and inflammation including Vascular Endothelial Growth Factors (VEGFs), Basic Fibroblast Growth Factors (FGF-2), and Platelet Derived Growth Factors (PDGFs), Insulin-Like GF (IGF1), Transforming GF-beta 1 (TGF- $\beta 1$ ), Vascular Endothelial GF (VEGF), Basic Fibroblastic GF (bFGF), and Epidermal GF (EGF) (3).

Growing evidence has suggested that the therapeutic efficacy of placental cells might depend on paracrine mechanisms, through the secretion of Extracellular vesicles (4). In this regard, small lipid-bound(30-150 nm), cellularly secreted vesicles that are called exosomes have especially attracted the attention of researchers (5). Exosomes are multivesicular bodies (MVBs) matured from early endosomes. They mediate local and systemic intercellular communication by fusing with target cells and releasing their macromolecular contents. Exosomes play an important role in many biological processes, including immune response and inflammation, blood coagulation, and angiogenesis $(6,7)$. Several studies have shown that exosomes derived from amniotic epithelial cells (AEC-Exo) improve wound healing and angiogenesis but their primary mechanisms remain unclear $(8,9)$.

In order to get a comprehensive understanding of the association between the angiogenic and wound healing effects of AEC-Exo treatments in current studies, we utilized a user-friendly bioinformatics analysis system. FunRich is a software with added options for functional enrichment and interaction network analyses of the genes and proteins (10). The Universal Protein Resource (UniProt) was examined via the Funrich tool to conduct enrichment analyses, including Gene ontology and protein domains. Moreover, in order to identify the proteins involved in degranulation and assess protein-protein association information, comparative and interaction network analyses were performed using various databases.

\section{Method}

\subsection{AEC-Exo proteins data}

The AEC-Exo proteins data of a proteomics analysis, deposited by Sheller et al. (2016), was downloaded from https://journals.plos.org/ (11). For further analyses, all protein ID reported in the study were converted to the UniProt protein identifier, and the repeated proteins were removed.

\subsection{Enrichment analyses}

The functional enrichment analyses of AEC-Exo proteins were performed using FunRich software version 3.1.3 available for public access. FunRich users are provided a user-friendly GUI (graphical user interface) allowing them to download directly from the FunRich database and UniProt, in order to further analyze their data. Therefore, a database from UniProt for human proteins (Taxon ID: 9606, ftp://ftp.uniprot.org/) was downloaded and the AEC-Exo proteins were analyzed against it. Several options were used for the enrichment analysis, including biological process, cellular component, molecular function, and protein domains. Subsequently, the results of the analyses were depicted graphically in the form of a column chart. 


\subsection{Comparative analyses}

In order to find the proteins involved in degranulation, Funrich was used to generate a Scalable-Venn diagram and Matrix table (also known as pair-wise comparison) among the total platelet degranulation proteins, the AECExo proteins, and the human exosomal proteins. One hundred and twenty-five platelet degranulation proteins were chosen in the NCBI BioSystems database via the Swiss-Prot/Uniprot filter, and all selected protein data was downloaded from the NCBI protein database. The human exosomal protein data was subsequently downloaded from ExoCarta (http://exocarta.org/download). Finally, to quantify the relative expression of proteins identified in the Human Proteome Map, the heatmap image was generated

\subsection{Interaction network analysis}

The STRING database (http://string-db.org/) was applied to illustrate and analyze protein-protein interaction (PPI) network. The interaction network analysis of FunRich was constructed for AEC-Exo proteins using UniProt as a background database.

\section{Result}

\subsection{AEC-Exo proteins and platelet degranulation}

Biological processes and Reactome pathway analysis indicated that $20.2 \%$ and $16.6 \%$ of the proteins in AECExo are remarkably engaged in platelet degranulation. ( $\mathrm{p}<0.001$, fold change $=17,22.1)$ (Figure 1,2$)$. In addition, $13.5 \%$ of these proteins are engaged in the regulation of Insulin-like growth factor (IGF) transport and its uptake by insulin-like growth factor-binding proteins (IGFBPs) (fold change $=11.7$ ).

A
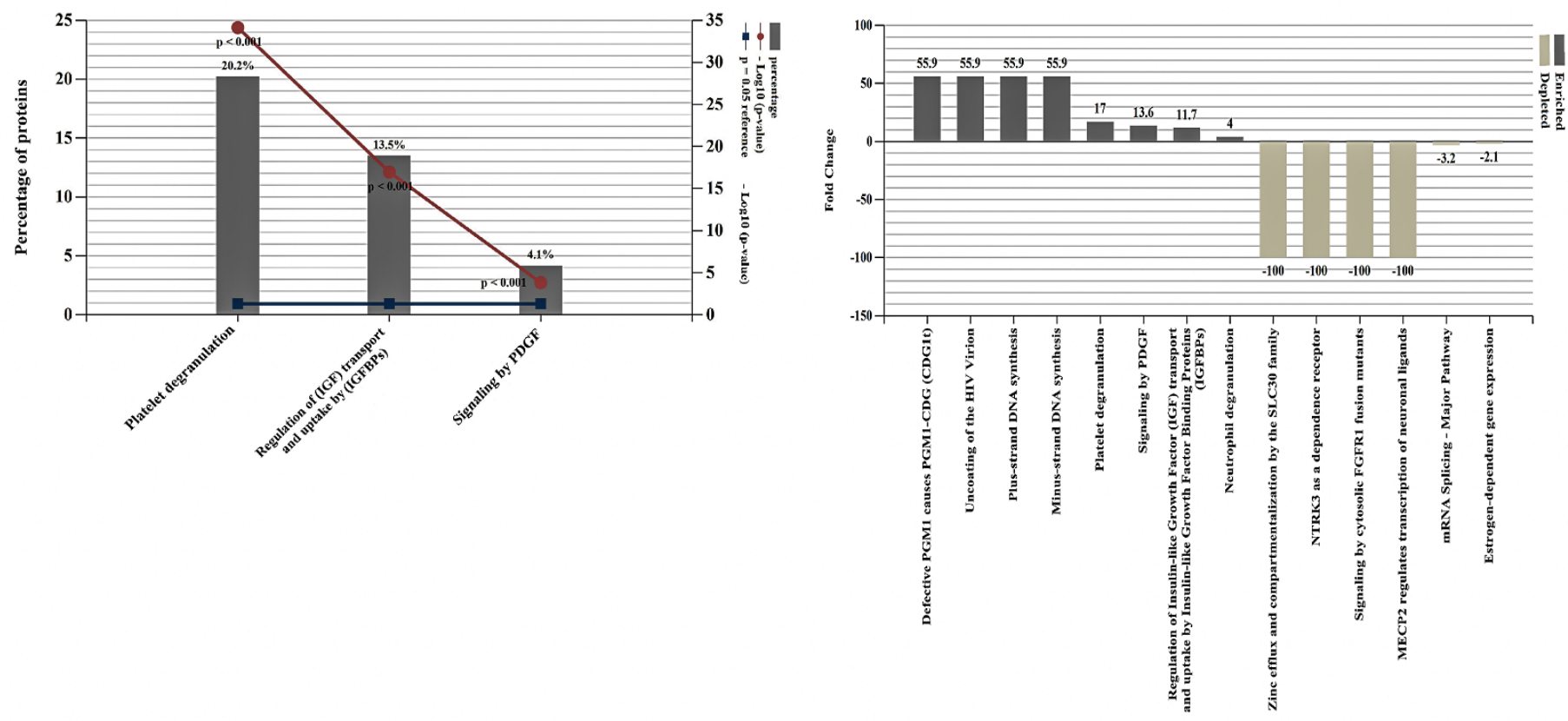

Figure 1: Reactome pathway analysis; $20.2 \%$ of the proteins in AEC-Exo are engaged in platelet degranulation ( $p<0.001$, fold change $=17$ ) 


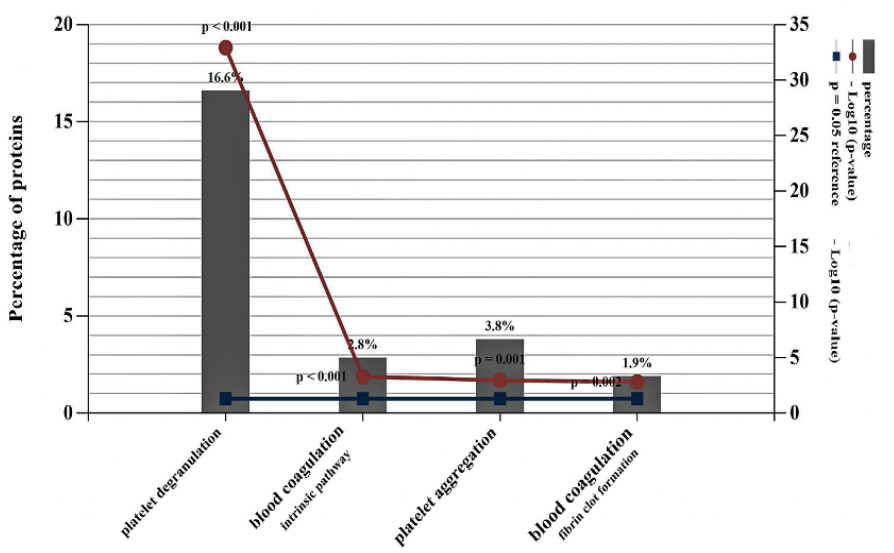

Biological process

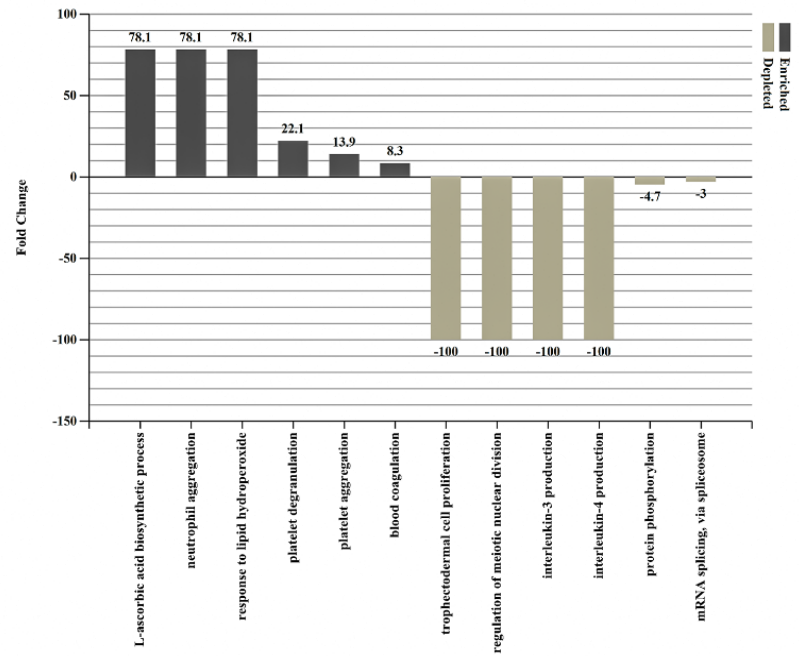

Figure 2: Biological process analysis; $16.6 \%$ of the proteins in AEC-Exo are involved in platelet degranulation ( $p<0.001$, fold change $=22.1$ )

\subsection{Protein domain, molecular function and cellular component analysis}

Protein domain analysis revealed the percentage of the proteins in AEC-Exo, including epidermal growth factor (EGF), calcium-binding EGF (EGF-CA), trypsin-like serine protease (Tryp-SPc), and serine proteinase inhibitors (SERPIN) (Fig. 3). The molecular function analysis of the proteins further revealed the involvement of calcium ion binding, platelet-derived growth factor binding and signaling receptor binding proteins (Fig. 4). The cellular component analysis of the proteins in AEC-Exo also showed the percentage of blood microparticle, platelet alpha granule lumen, platelet dense granule lumen and platelet alpha granule proteins $(\mathrm{p}<0.001)$ (Fig. 5).
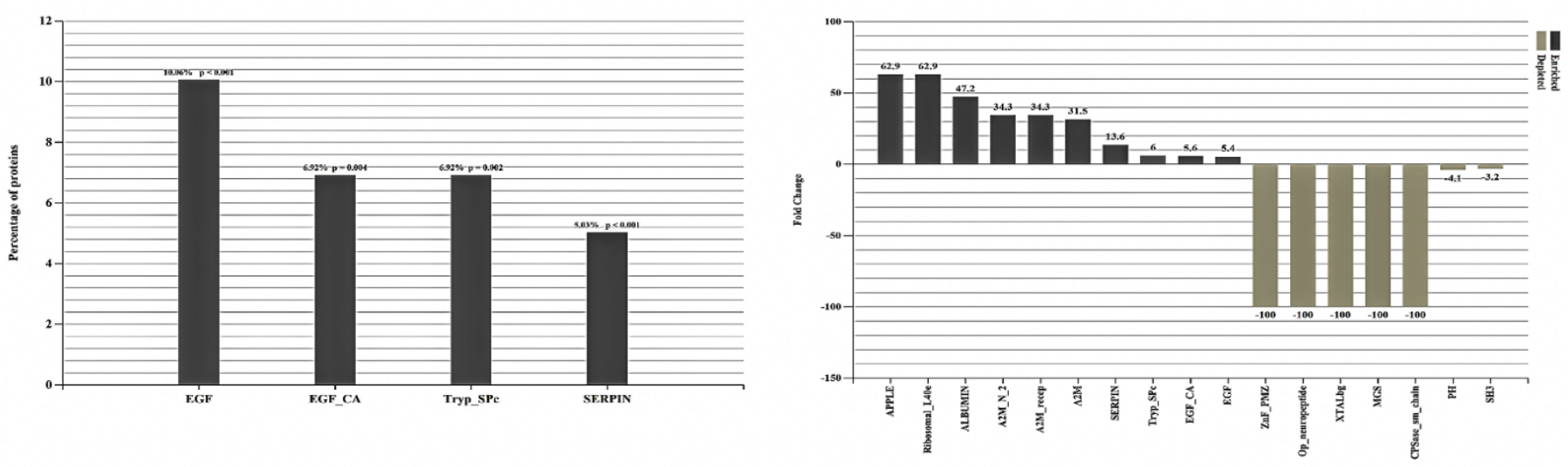

Figure 3: (A) Protein domain analysis of the proteins in AEC-Exo ( $p<0.001)$

(B) Separate number of fold enriched/depleted of every protein domain 

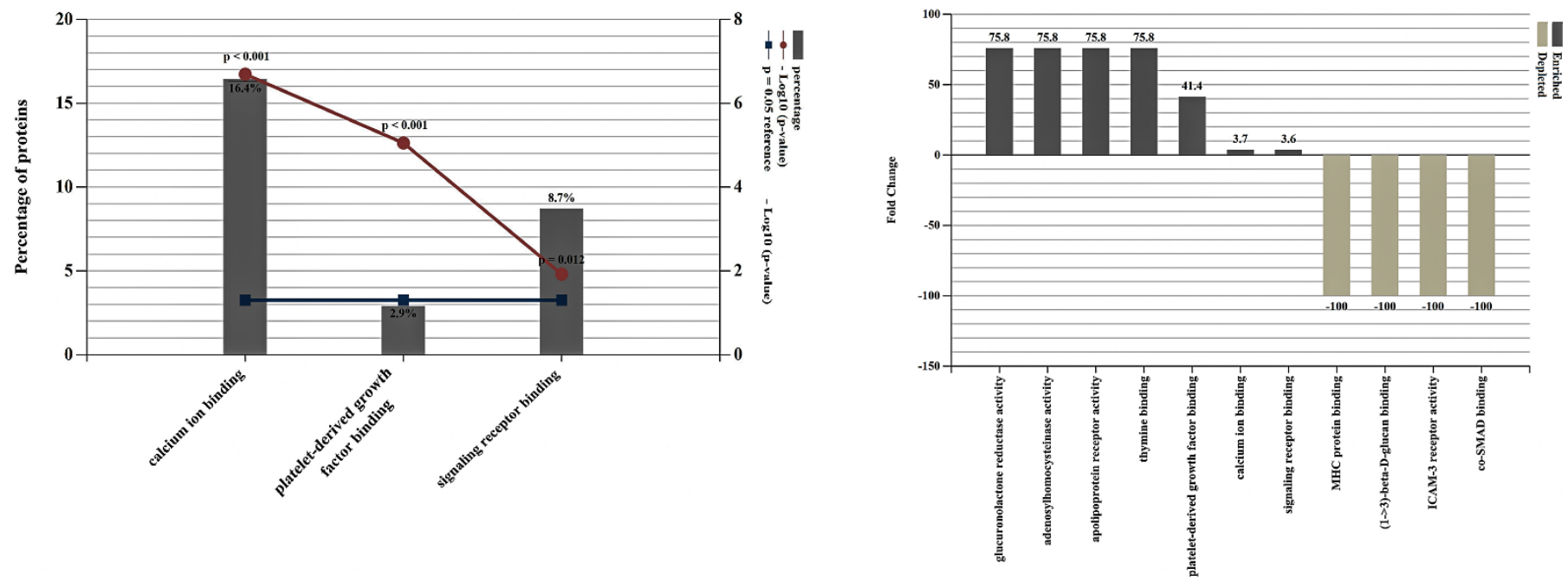

Figure 4: (A) Molecular function analysis of the AEC-Exo proteins

(B) Separate number of fold enriched/depleted of each molecular function

$\mathbf{A}$

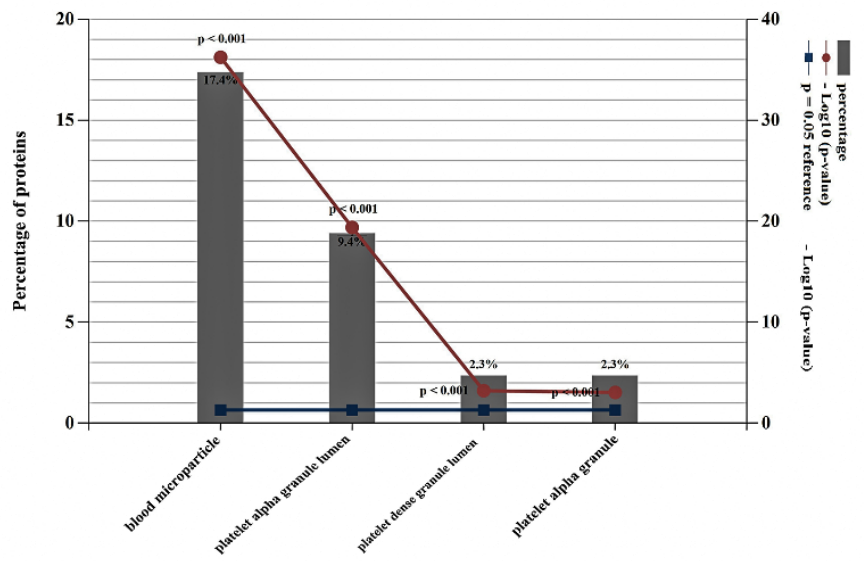

Cellular component

B

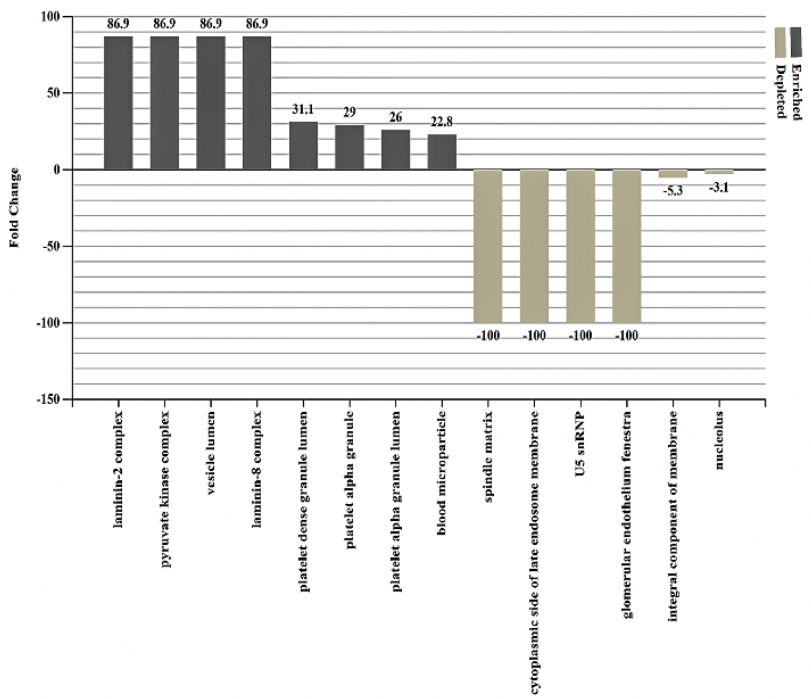

Figure 5: (A) Cellular component analysis of the AEC-Exo proteins

(B) Separate number of fold enriched/depleted of cellular component 


\subsection{Protein identification and comparison analysis}

Thirty-seven proteins were identified that were involved in platelet degranulation in the AEC-Exo (Fig. 6, Table 1). The Venn diagram and the Matrix table revealed the overlap of 103 proteins between human exosomal proteins, and total platelet degranulation proteins. Figure 7 illustrated the heatmap image of AEC-Exo proteins involved in platelet degranulation based on the human proteome map.

A

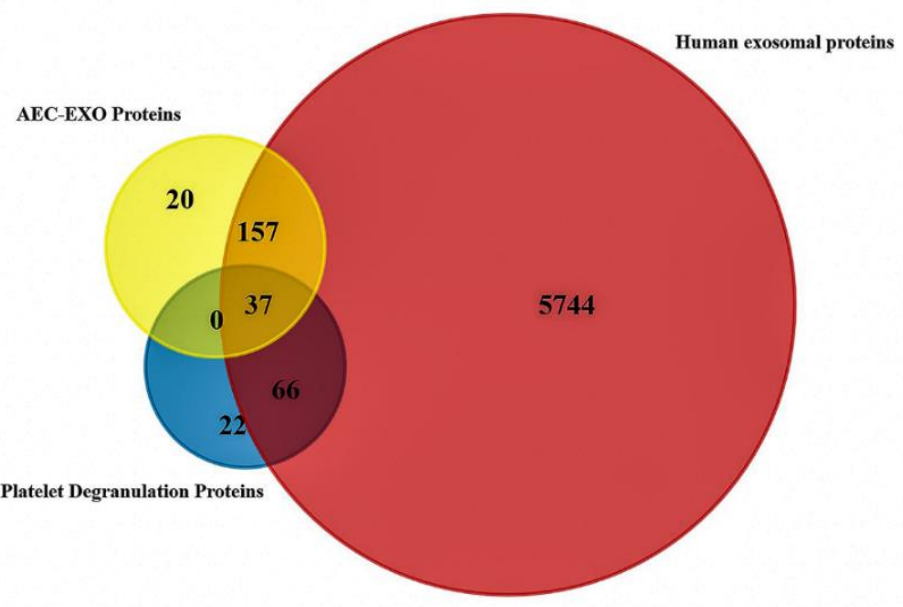

B

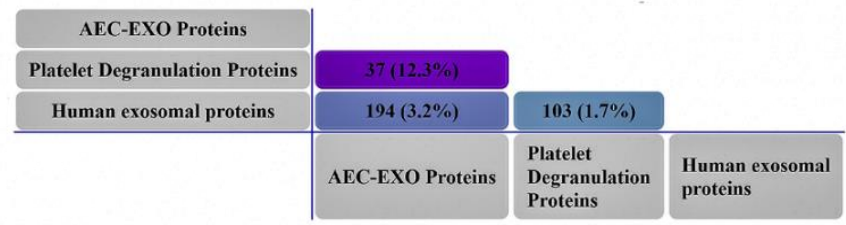

Figure 6: (A) Venn diagram showing the overlaps between total platele degranulation proteins, human exosomal proteins and AEC-Exo. (B) Matrix table (pair-wise comparison) for AEC-Exo, total platelet degranulation proteins and human exosomal proteins. 
Table 1: The List of the proteins in AEC-Exo involved in platelet degranulation

\begin{tabular}{|c|c|c|}
\hline Identifier & Protein names & Length (aa) \\
\hline A2M & Alpha-2-macroglobulin & 1474 \\
\hline ACTN1 & Alpha-actinin-1 & 914 \\
\hline ACTN4 & Alpha-actinin-4 & 911 \\
\hline ALDOA & Fructose-bisphosphate aldolase A & 418 \\
\hline APOA1 & Apolipoprotein A-I & 267 \\
\hline APOH & Beta-2-glycoprotein 1 & 345 \\
\hline CAP1 & Adenylyl cyclase-associated protein 1 & 475 \\
\hline CD109 & CD109 antigen & 1445 \\
\hline CLEC3B & Tetranectin & 202 \\
\hline CLU & Clusterin & 449 \\
\hline Cofilin-1 & Cofilin-1 & 166 \\
\hline F13A1 & Coagulation factor XIII A chain & 732 \\
\hline F5 & Coagulation factor $\mathrm{V}$ & 2224 \\
\hline FERMT3 & Fermitin family homolog 3 & 667 \\
\hline FGB & Fibrinogen beta chain & 491 \\
\hline FGG & Fibrinogen gamma chain & 453 \\
\hline FLNA & Filamin-A & 2647 \\
\hline IGF2 & Insulin-like growth factor II & 236 \\
\hline ITIH3 & Inter-alpha-trypsin inhibitor heavy chain $\mathrm{H} 3$ & 890 \\
\hline ITIH4 & Inter-alpha-trypsin inhibitor heavy chain $\mathrm{H} 4$ & 930 \\
\hline PFN1 & Profilin-1 & 140 \\
\hline PLG & Plasminogen & 810 \\
\hline PPIA & Peptidyl-prolyl cis-trans isomerase A & 165 \\
\hline PROS1 & Vitamin K-dependent protein S & 676 \\
\hline QSOX1 & Sulfhydryl oxidase 1 & 747 \\
\hline SERPINE1 & Plasminogen activator inhibitor 1 & 402 \\
\hline SERPINF2 & Alpha-2-antiplasmin & 491 \\
\hline TAGLN2 & Transgelin-2 & 220 \\
\hline TF & Serotransferrin & 698 \\
\hline THBS1 & Thrombospondin-1 & 1170 \\
\hline TIMP3 & Metalloproteinase inhibitor 3 & 211 \\
\hline TLN1 & Talin-1 & 2541 \\
\hline TTN & Titin & 35991 \\
\hline TUBA4A & Tubulin alpha- $4 \mathrm{~A}$ chain & 448 \\
\hline VCL & Vinculin & 1134 \\
\hline VWF & Von Willebrand factor & 2813 \\
\hline WDR1 & WD repeat-containing protein 1 & 606 \\
\hline
\end{tabular}

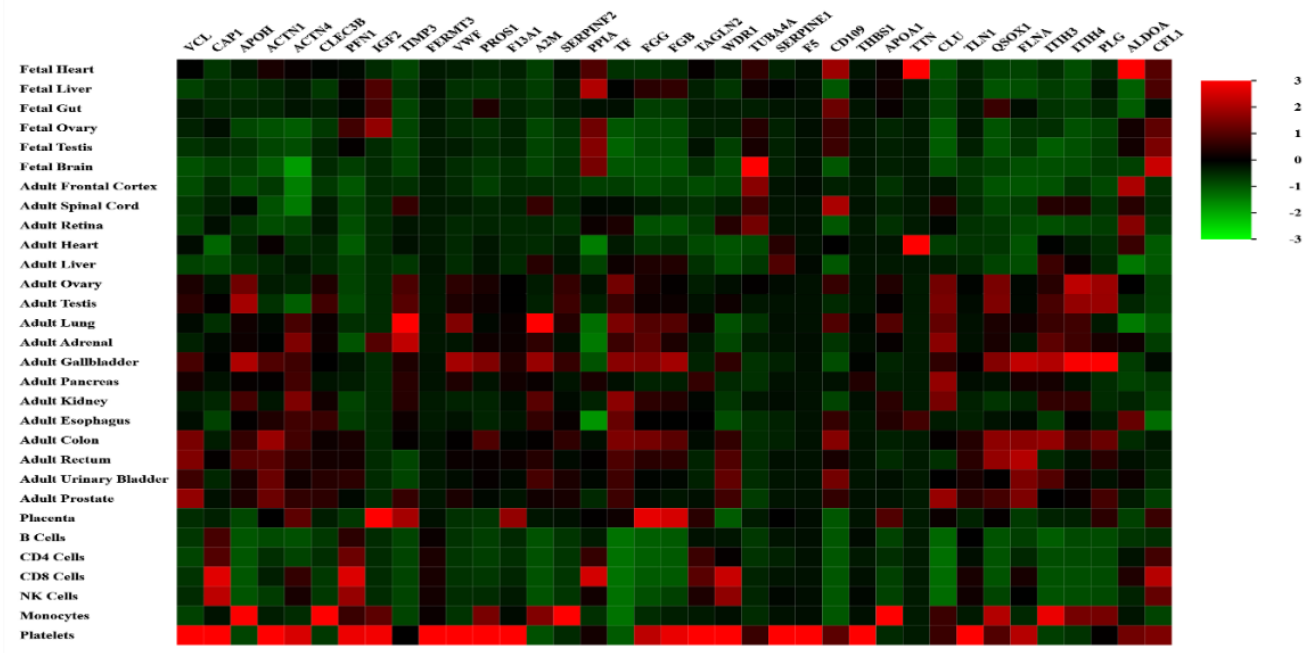

Figure 7: Heat map representing of the proteins in AEC-Exo involved in platelet degranulation (green - lowest abundance and red — highest abundance) 


\subsection{Predicting PPI network of platelet degranulation}

To investigate the interactions between the proteins that participated in platelet degranulation, a PPI network was constructed. The interaction network planetary layout of AEC-EXO proteome data via the FunRich displayed 39 genes significantly enriched for platelet degranulation $(\mathrm{p}<0.001)$ (Fig. 8). Fibronectin (FN1) with the highest degree of distribution is placed in the center of the network construction. On the other hand, the number of 259 edges through the STRING represents protein-protein associations that are significant, therefore the proteins contribute to a shared function equally (PPI enrichment p-value < 1.0e-16) (Fig. 9).

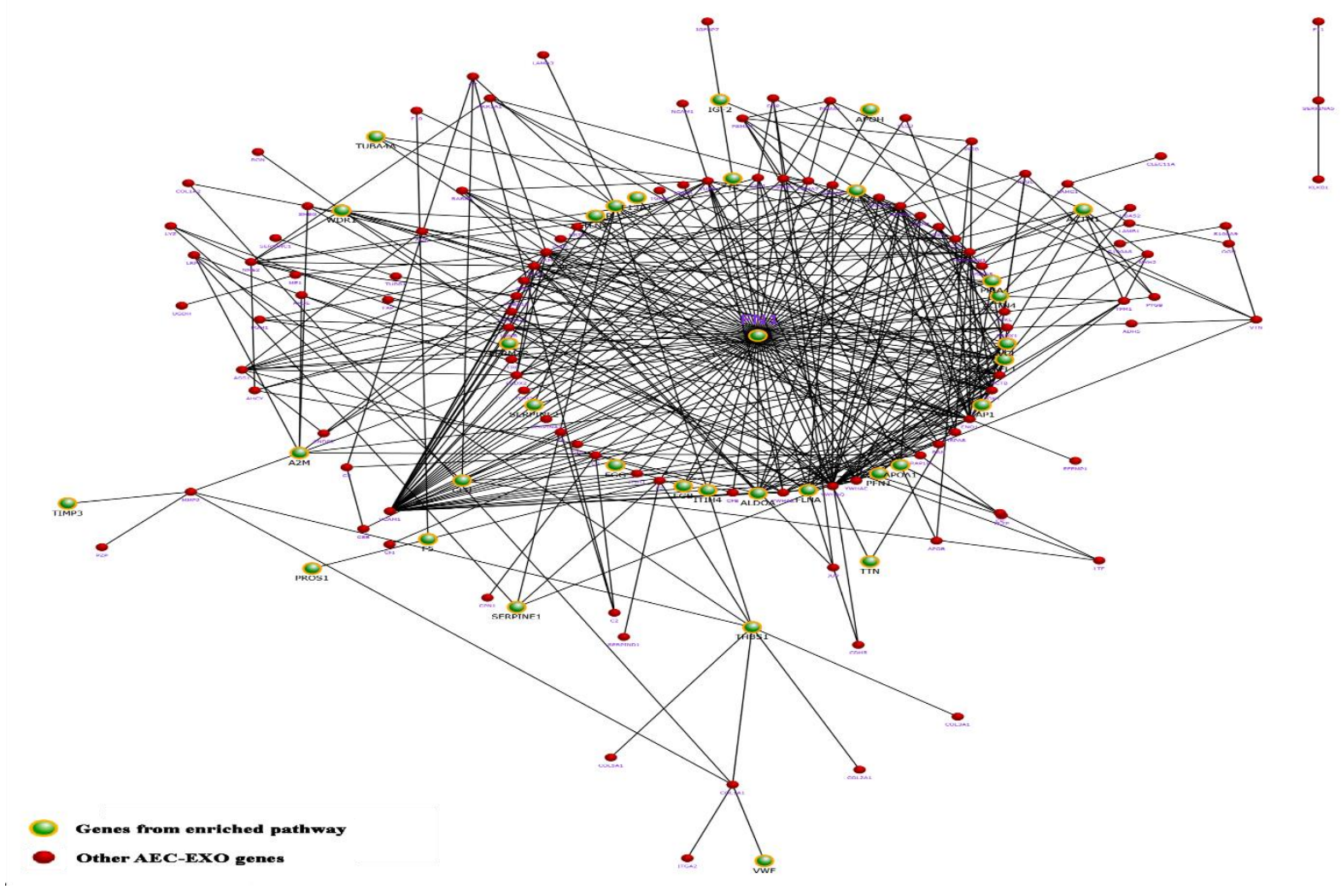

Figure 8: Interaction network planetary layout of AEC-Exo proteome data via the FunRich $(p<0.001)$. The list of genes enriched in platelet degranulation were highlighted within the interactions network. 


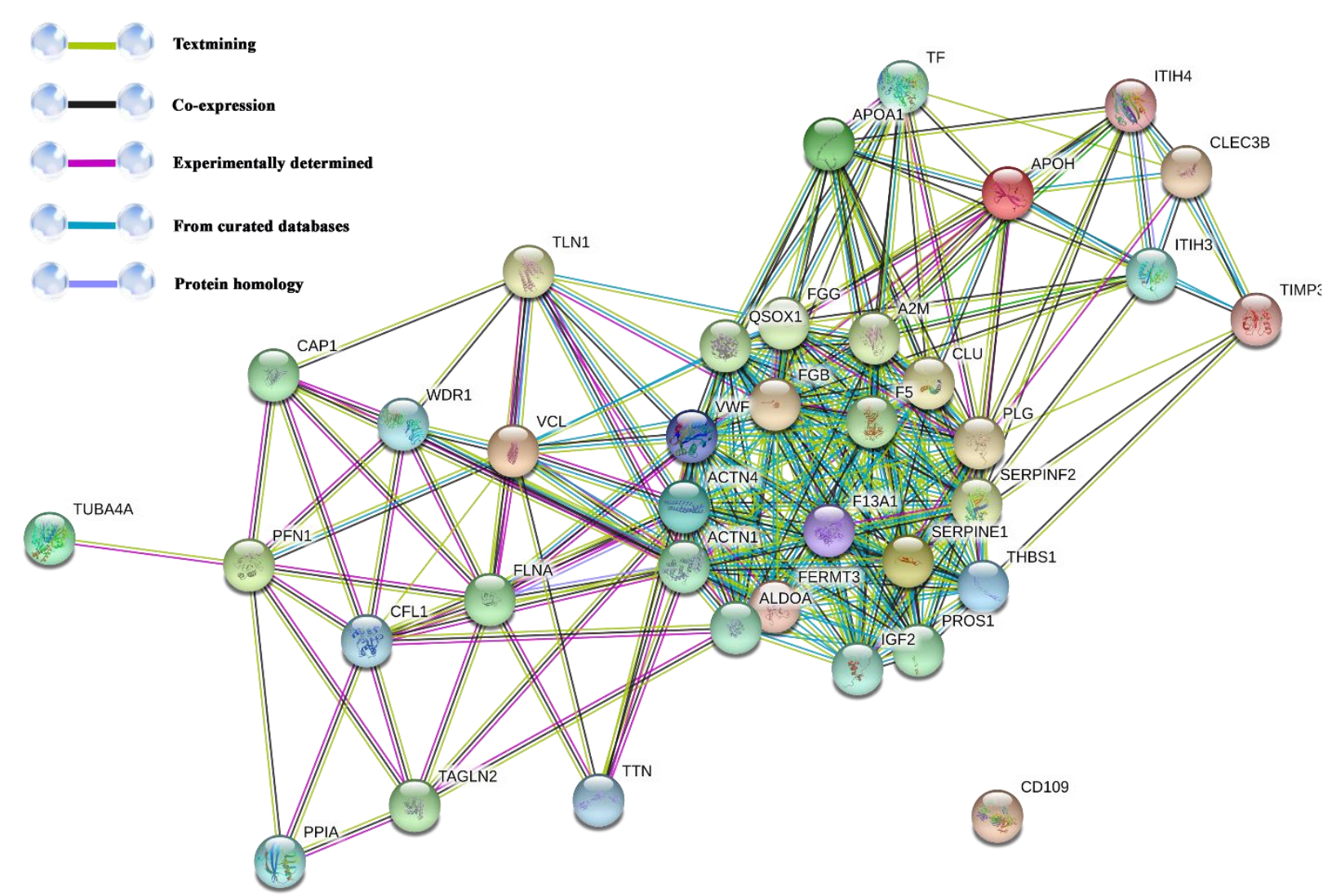

Figure 9: PPI network analysis with the STRING database of the 37 proteins in AEC-Exo involved in platelet degranulation. The edges represent protein-protein associations. (PPI enrichment p-value $<1.0 \mathrm{e}-16$ )

\section{Discussion}

The results of the present investigation demonstrate the significant association between AEC-Exo proteome, platelet degranulation, and their corresponding processes. A significant relevance was observed between the proteins in AEC-Exo, and platelet degranulation which is pivotal as a primary mechanism for the healing process and angiogenesis. In addition, interaction network analysis using the STRING and FunRich validated enrichment in the platelet degranulation. Over the past decades, efforts have been made to modulate angiogenesis as a therapeutic strategy to either increase revascularization of ischemic tissues or inhibit angiogenesis in cancer, ocular, joint, or skin disorders. Consistent with the literature, other studies have confirmed AEC-Exo and placental exosome's role in increasing wound healing and angiogenesis (12-14). Consequently, the involvement of platelet degranulation in AEC-Exo proteins may illustrate the angiogenic effects of AEC-Exo treatments in current studies.

The results of protein domain analysis showed that the highest protein percentage belongs to EGF domain. Each EGF domain is comprised about 40 to 45 amino acids and found in a large variety of proteins. Most of the angiogenic factors contain the EGF-like domain, which may exhibit a mitogenic effect on endothelial cells and facilitate neovascularization $(15,16)$. For instance, Kao YC et al. (2012) demonstrated that the EGF-like domain is essential for CD93 to induce angiogenesis (17). Hence, the inclusion of these domains in the AEC-EXO proteins increasingly affects the modulation of angiogenesis and wound healing. The Reactome pathway analysis revealed the importance of the IGFBP family in the AEC-EXO proteins. It has been shown that they have both stimulate 
and inhibit angiogenesis (18). Activation of VEGF is modulated by IGFBP-2 to induce an angiogenic-type response in human umbilical vein endothelial cells (HUVEC) in vitro. Studies have shown IGF1 stimulates endothelial cell sprouting in an in vitro assay. PDGF has an important role in angiogenesis but molecular component analysis of the proteins in AEC-Exo only showed four percent participation (19-21).

Based on molecular function analysis conducted in this study, the proteins in AEC-Exo remarkably participated in calcium ion binding, platelet-derived growth factor binding, and signaling receptor binding. Bioactive molecules of dense granules containing catecholamines, histamine, serotonin, ADP, ATP, calcium ions, and dopamine, which are active in vasoconstriction, increased capillary permeability, attracting and activating macrophages, tissue modulation, and regeneration. The secretion of PDGFB, TGF-beta1, bFGF, and VEGF is considerably mediated by the amount of calcium and thrombin (Thr) added to the platelet-rich plasma (PRP), and PRP supernatants are more mitogenic for endothelial cells than whole-blood supernatants. Endothelial cells are crucial in vessel formation by angiogenesis and vasculogenesis. Intracellular calcium signals are involved in different critical phases in the regulation of the complex and multi-stepped process of angiogenesis. Furthermore, regarding either external activation with calcium and thrombin or internal activation with endogenous tissue thromboplastin, the fibrin matrix forms by polymerization of plasma fibrinogen (22-24). Accordingly, our observations further support and strengthen the importance of the AEC-Exo in angiogenesis and wound healing by platelet degranulation.

\section{Conclusion}

Based on these findings, the involvement of platelet degranulation in AEC-Exo proteins may elucidate the angiogenic and wound healing effects of AEC-Exo treatments in current studies. Collectively, our bioinformaticbased study provided the AEC-EXO proteome, which can be of high interest to reveal biological insights about the healing process and angiogenesis.

\section{References}

1. Rozman P, Bolta Z. Use of platelet growth factors in treating wounds and soft-tissue injuries. Acta Dermatovenerologica Alpina Panonica et Adriatica. 2007;16(4):156.

2. Li J, Zhang YP, Kirsner RS. Angiogenesis in wound repair: angiogenic growth factors and the extracellular matrix. Microscopy research and technique. 2003;60(1):107-14.

3. Martínez CE, Smith PC, Palma Alvarado VA. The influence of platelet-derived products on angiogenesis and tissue repair: a concise update. Frontiers in physiology. 2015;6:290.

4. $\quad$ Song Y-S, Joo H-W, Park I-H, Shen G-Y, Lee Y, Shin JH, et al. Transplanted Human Amniotic Epithelial Cells Secrete Paracrine Proangiogenic Cytokines in Rat Model of Myocardial Infarctio. Cell transplantation. 2015;24(10):2055-64.

5. Komaki M, Numata Y, Morioka C, Honda I, Tooi M, Yokoyama N, et al. Exosomes of human placentaderived mesenchymal stem cells stimulate angiogenesis. Stem cell research \& therapy. 2017;8(1):219.

6. Fernandes Ribeiro M, Zhu H, W Millard R, Fan G-C. Exosomes function in pro-and anti-angiogenesis. Current angiogenesis. 2013;2(1):54-9.

7. Zhang Y, Liu Y, Liu H, Tang WH. Exosomes: biogenesis, biologic function and clinical potential. Cell \& bioscience. 2019;9(1):19.

8. Zhao B, Li X, Shi X, Shi X, Zhang W, Wu G, et al. Exosomal microRNAs derived from human amniotic epithelial cells accelerate wound healing by promoting the proliferation and migration of fibroblasts. Stem cells international. 2018;2018.

9. Zhao B, Zhang Y, Han S, Zhang W, Zhou Q, Guan H, et al. Exosomes derived from human amniotic epithelial cells accelerate wound healing and inhibit scar formation. Journal of molecular histology. 2017;48(2):121-32. 
10. Pathan M, Keerthikumar S, Ang CS, Gangoda L, Quek CY, Williamson NA, et al. FunRich: An open access standalone functional enrichment and interaction network analysis tool. Proteomics. 2015;15(15):2597601.

11. Sheller S, Papaconstantinou J, Urrabaz-Garza R, Richardson L, Saade G, Salomon C, et al. Amnionepithelial-cell-derived exosomes demonstrate physiologic state of cell under oxidative stress. PLoS One. 2016;11(6).

12. Harp D, Driss A, Mehrabi S, Chowdhury I, Xu W, Liu D, et al. Exosomes derived from endometriotic stromal cells have enhanced angiogenic effects in vitro. Cell and tissue research. 2016;365(1):187-96.

13. Tan JL, Lau SN, Leaw B, Nguyen HP, Salamonsen LA, Saad MI, et al. Amnion epithelial cell-derived exosomes restrict lung injury and enhance endogenous lung repair. Stem cells translational medicine. 2018;7(2):180-96.

14. Bian X, Ma K, Zhang C, Fu X. Therapeutic angiogenesis using stem cell-derived extracellular vesicles: an emerging approach for treatment of ischemic diseases. Stem cell research \& therapy. 2019;10(1):158.

15. Fréchette J-P, Martineau I, Gagnon G. Platelet-rich plasmas: growth factor content and roles in wound healing. Journal of dental research. 2005;84(5):434-9.

16. Froum SJ, Wallace SS, Tarnow DP, Cho S-C. Effect of platelet-rich plasma on bone growth and osseointegration in human maxillary sinus grafts: three bilateral case reports. International Journal of Periodontics and Restorative Dentistry. 2002;22(1):45-54.

17. Kao Y-C, Jiang S-J, Pan W-A, Wang K-C, Chen P-K, Wei H-J, et al. The epidermal growth factor-like domain of CD93 is a potent angiogenic factor. PloS one. 2012;7(12).

18. Slater T, Haywood NJ, Matthews C, Cheema H, Wheatcroft SB. Insulin-like growth factor binding proteins and angiogenesis: from cancer to cardiovascular disease. Cytokine \& growth factor reviews. 2019;46:2835 .

19. Bach LA. Endothelial cells and the IGF system. J Mol Endocrinol. 2015;54(1):R1-R13.

20. Boswell EJ, Kurniawan ND, Downing AK. Calcium-binding EGF-like domains. Encyclopedia of Inorganic and Bioinorganic Chemistry. 2011.

21. Jacobo SMP, Kazlauskas A. Insulin-like growth factor 1 (IGF-1) stabilizes nascent blood vessels. Journal of Biological Chemistry. 2015;290(10):6349-60.

22. Sánchez-González DJ, Méndez-Bolaina E, Trejo-Bahena NI. Platelet-rich plasma peptides: key for regeneration. International journal of peptides. 2012;2012.

23. Martineau I, Lacoste E, Gagnon G. Effects of calcium and thrombin on growth factor release from platelet concentrates: kinetics and regulation of endothelial cell proliferation. Biomaterials. 2004;25(18):4489-502.

24. Munaron L. Intracellular calcium, endothelial cells and angiogenesis. Recent patents on anti-cancer drug discovery. 2006;1(1):105-19. 